\title{
BLOCKING SETS IN DESARGUESIAN PROJECTIVE PLANES
}

\author{
A. BLOKHUIS AND A. E. BROUWER
}

\begin{abstract}
Using theorems of Redéi, and of Brouwer and Schrijver, and Jamison, it is proved that a non-trivial blocking set in a desarguesian projective plane of order $q$ has at least $q+\sqrt{ }(2 q)+1$ points, if $q$ is at least 7 , odd and not a square and $q \neq 27$. Further one can show that non-trivial blocking sets in the desarguesian planes $P G(2,11)$ and $P G(2,13)$ have at least 18 resp. 21 points, and this is best possible. In addition a nice description of a blocking set of size $q^{t}+q^{t-1}+1$ in the desarguesian plane $\operatorname{PG}\left(2, q^{t}\right)$ is given, where $q$ is some prime power.
\end{abstract}

\section{Introduction}

A blocking set in a linear space is a set $S$ of points, such that each line intersects $S$ in at least one point. $S$ is called non-trivial, if no line is completely contained in $S$, in the case of a projective plane. In this note we want to derive lower bounds for the cardinality of $S$.

\section{Two useful theorems}

The following construction yields interesting blocking sets in the desarguesian plane $\mathrm{PG}(2, q)$ :

Let $f: \mathrm{GF}(q) \rightarrow \mathrm{GF}(q)$ be any non-linear function. Form a blocking set consisting of

(i) the $q$ points forming the graph of $f$ in $\operatorname{AG}(2, q)$,

(ii) the directions determined by $f$ on the line at infinity, say $m$ points.

EXAMPLE 1. Let $q=p$ be a prime, $f(x)=x^{\frac{1}{2}(p+1)}$. This yields a blocking set of $\frac{3}{2}(p+1)$ points, which is conjectured to be best possible ([7], see also [6]).

2. Let $q=q_{1}^{t}(t>1)$, then $\operatorname{GF}\left(q_{1}\right)$ is a subfield of $\mathrm{GF}(q)$. Let $f$ be the trace map from $\operatorname{GF}(q)$ to $\operatorname{GF}\left(q_{1}\right)$. Then $S=q+q / q_{1}+1$. This is also the best known, if $q_{1}$ is chosen maximal (compare $[\mathbf{2}, \mathbf{4}]$ ).

The following theorem gives lower bounds for $m$, where $q=p^{n}, p$ prime.

THEOREM. ([8, p. 237], see also [6]).

$$
m \geqslant \frac{q-1}{p^{\frac{1}{2}} n+1}+1, \text { and } m \geqslant \frac{p+3}{2} \text { if } n=1 \text {. }
$$

COROLlaRY. Let $X$ be a set of $q$ points in the desarguesian affine plane of order $q$, determining $m$ directions. Then $m$ satisfies the above inequalities.

Proof. Either $X$ determines all directions, or there is a parallel class all of whose lines contain exactly 1 point of $X$.

Received I June 1984.

1980 Mathematics Subject Classification 05B25.

Bull. London. Math. Soc. 18 (1986) 132-134 
Let $S$ be a minimal blocking set; then each point of $S$ is on at least one tangent. Let $p \in S$ be a point on $t$ tangents, call one tangent $l$, and form a blocking set of $\operatorname{AG}(2, q)=\mathrm{PG}(2, q) \backslash l$ with $|S|-1+t-1$ points in the obvious way.

THEOREM $([1,5])$. A blocking set of a desarguesian affine plane $\mathrm{AG}(2, q)$ has at least $2 q-1$ points.

As a consequence of this, one has that $t \geqslant 2 q+1-|S|$ for each point in $S$. Using these two results it is now a trivial exercise to show that a blocking set in the desarguesian plane $\operatorname{PG}(2,11)$ has at last 18 points, and a rather tedious one to prove $|S| \geqslant 21$ for $\mathrm{PG}(2,13)$.

\section{Blocking sets in the desarguesian plane $\mathrm{PG}(2, q)$}

It is well known, and due to Bruen [2], that $|S| \geqslant q+\sqrt{ } q+1$, with equality if and only if $q$ is a square and $S$ a Baer-subplane. When $q$ is not a square this bound can be improved.

Let $S$ be a blocking set of size $|S|=q+m$. If $S$ contains an $m$-secant the corollary gives a lower bound for the cardinality of $S$. The next theorem treats the remaining case.

THEOREM. Let $S$ be a blocking set of size $q+m$ without an $m$-secant. Then

$$
|S| \geqslant q+\sqrt{ }(2 q)+1 \text {. }
$$

Proof. Since each line contains at most $m-1$ points of $S$, it follows that each point is on at most $q-1$ tangents. Counting incident pairs (tangent, point not in $S$ ) in two ways, using the second theorem, one gets

or, rewriting,

$$
q(q+m)(q-m+1) \leqslant\left(q^{2}-m+1\right)(q-1)
$$

$$
2 q \leqslant(m-1)^{2}+(m-1) / q, \quad \text { whence } m \geqslant \sqrt{ }(2 q)+1 \text {. }
$$

Corollary. Suppose $q$ is odd, not a square, at least 7 and not 27. Let $S$ be an arbitrary non-trivial blocking set of the desarguesian plane $\operatorname{PG}(2, q)$. Then $|S| \leqslant q+\sqrt{ }(2 q)+1$.

\section{Final remarks}

If $q<7$ everything is known; if $q=27$ we only get $|S| \geqslant 35$; if $q=2^{2 t+1}$ one obtains $|S| \geqslant 2^{2 t+1}+2^{t+1}$.

The first paper relating Redéi's theorem to blocking sets seems to be [3]. We wonder whether Redéi's theorem can be improved to $m \geqslant 1+q / q_{1}$, where $q_{1}$ is the order of maximal subfield of $\mathrm{GF}(q)$.

\section{References}

1. A. E. BrouWER and A. SCHRIJVER, 'The blocking number of an affine space', J. Combin. Theory Ser. A 24 (1978) 251-253.

2. A. A. BrUEN, 'Blocking sets in finite projective planes', SIAM J. Appl. Math. 21 (1971) 380-392. 
3. A. A. Bruen and J. A. Thas, 'Blocking sets', Geom. Dedicata 6 (1977) 193-203.

4. J. W. P. HirsCHFen, Projective geometries over finite fields (Oxford University Press, 1979).

5. R. JAMISON, 'Covering finite fields with cosets of subspaces', J. Combin. Theory Ser. A 22 (1977) 253-266.

6. L. LovasZ and A. SCHRIJver, 'Remarks on a theorem of Redéi', Studia Sci. Math. Hungar. 16 (1981) 449-454.

7. J. DI PaOla, 'On a restricted class of block design games', Canad. J. Math. 18 (1966) 225-236.

8. L. ReDÉ, Lückenhafte Polynome über endlichen Körpern (Birkhäuser Verlag, Basel, 1970).

Techn. University Eindhoven

P.O. Box 513

5600 MB Eindhoven

Netherlands
C.W.I.

Kruislaan 413

1098 SJ Amsterdam

Netherlands 